ARTICLE

\title{
Strong optomechanical coupling at room temperature by coherent scattering
}

Andrés de los Ríos Sommer ${ }^{1}$, Nadine Meyer (iD) ${ }^{1 凶}$ \& Romain Quidant (1D) $1,2,3$

Quantum control of a system requires the manipulation of quantum states faster than any decoherence rate. For mesoscopic systems, this has so far only been reached by few cryogenic systems. An important milestone towards quantum control is the so-called strong coupling regime, which in cavity optomechanics corresponds to an optomechanical coupling strength larger than cavity decay rate and mechanical damping. Here, we demonstrate the strong coupling regime at room temperature between a levitated silica particle and a high finesse optical cavity. Normal mode splitting is achieved by employing coherent scattering, instead of directly driving the cavity. The coupling strength achieved here approaches three times the cavity linewidth, crossing deep into the strong coupling regime. Entering the strong coupling regime is an essential step towards quantum control with mesoscopic objects at room temperature.

\footnotetext{
${ }^{1}$ ICFO-Institut de Ciencies Fotoniques, The Barcelona Institute of Science and Technology, 08860 Castelldefels (Barcelona), Spain. ${ }^{2}$ ICREA-Institució Catalana de Recerca i Estudis Avançats, 08010 Barcelona, Spain. ${ }^{3}$ Nanophotonic Systems Laboratory, Department of Mechanical and Process Engineering,

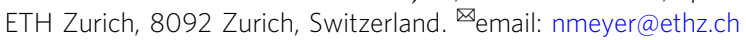


aser cooling has revolutionised our understanding of atoms, ions and molecules. Lately, after a decade of experimental and theoretical efforts employing the same techniques ${ }^{1-8}$, the motional ground state of levitated silica nanoparticles at room temperature has been reported ${ }^{9}$. While this represents an important milestone towards the creation of mesoscopic quantum objects, coherent quantum control of levitated nanoparticles ${ }^{10,11}$ still remains elusive.

Levitated particles stand out among the plethora of optomechanical systems ${ }^{12}$ due to their detachment, and therefore high degree of isolation from the environment. Their centre of mass, rotational and vibrational degrees of freedom ${ }^{13}$ make them attractive tools for inertial sensing ${ }^{14}$, rotational dynamics ${ }^{15-18}$, free fall experiments ${ }^{19}$, exploration of dynamic potentials ${ }^{20}$, and are envisioned for testing macroscopic quantum phenomena at room temperature ${ }^{2,10,21,22}$.

Recently, the centre-of-mass motion of a levitated particle has successfully been $3 \mathrm{D}$ cooled employing coherent scattering $(\mathrm{CS})^{8,23}$. Cooling with CS is less sensitive to phase noise heating than actively driving the cavity 7,24 , because optimal coupling takes place at the intensity node. Lately, this has enabled phonon occupation numbers of less than one ${ }^{9}$.

For controlled quantum experiments, such as the preparation of non-classical, squeezed ${ }^{25,26}$ or entangled states ${ }^{27,28}$, the particle's motional state needs to be manipulated faster than the absorption of a single phonon from the environment. A valuable but less stringent condition is the so-called strong coupling regime (SCR), where the optomechanical coupling strength $g$ between the mechanical motion of a particle and an external optical cavity exceeds the particle's mechanical damping $\Gamma_{\mathrm{m}}$ and the cavity linewidth $\kappa\left(g \gg \Gamma_{\mathrm{m}}, \kappa\right)$. The SCR presents one of the first stepping stones towards full quantum control and has been demonstrated in opto- and electromechanical systems ${ }^{29-31}$, followed by quantum-coherent control ${ }^{32}$.

Here, we observe normal mode splitting (NMS) in SCR with levitated nanoparticles ${ }^{33}$, as originally reported in atoms ${ }^{34}$. In contrast to previous experiments, we employ $\mathrm{CS}^{8,23,35,36}$. Our table-top experiment offers numerous ways to tune the optomechanical coupling strength at room temperature, a working regime that is otherwise nearly exclusive to plasmonic nanocavities 37,38 .

\section{Results}

Experimental setup for levitation. Our experimental setup is displayed in Fig. 1. A silica nanoparticle (green) of radius $R \approx 90 \mathrm{~nm}$, mass $m=6.4 \times 10^{-18} \mathrm{~kg}$ and refractive index $n_{\mathrm{r}}=1.45$ is placed in a cavity (purple) by an optical tweezers trap (yellow) with wavelength $\lambda_{\mathrm{t}}=2 \pi / k_{\mathrm{t}}=1064 \mathrm{~nm}$, power $P_{\mathrm{t}} \simeq 150 \mathrm{~mW}$, numerical aperture $\mathrm{NA}=0.8$, and optical axis $(z)$ perpendicular to the cavity axis $(y)$. The trap is linearly polarised along the axis defined as $\epsilon_{\theta}=\epsilon_{x} \cos \theta$ (see inset in Fig. 1).

The nanoparticle's eigenfrequencies $\Omega_{x, y, z}=2 \pi \times(172 \mathrm{kHz}$, $197 \mathrm{kHz}, 56 \mathrm{kHz}$ ) are non-degenerate due to tight focusing. The trap is mounted on a nano-positioning stage allowing for precise $3 \mathrm{D}$ placement of the particle inside the low loss, high finesse Fabry-Pérot cavity with a cavity linewidth $\kappa \approx 2 \pi \times 10 \mathrm{kHz}$, cavity finesse $F=5.4 \times 10^{5}$ and free spectral range $\Delta \omega_{\mathrm{FSR}}=\pi c / L_{\mathrm{c}}=$ $2 \pi \times 5.4 \mathrm{GHz}$. The relative detuning $\Delta=\omega_{\mathrm{t}}-\omega_{\mathrm{c}}$ between the trap and the cavity resonance is tunable. The intracavity photon number $n_{\text {cav }}$ is estimated from the transmitted cavity power $P_{\text {out }}$ ( $\mathrm{CO}$ in Fig. 1), and the particle position displacement is measured by interfering the scattered light with a co-propagating reference beam $^{39}$. In CS, scattering events from the detuned trapping field, locked at $\Delta$, populate the cavity. This contrasts the approach of actively driving the cavity $3,7,24$. A particle in free space, solely

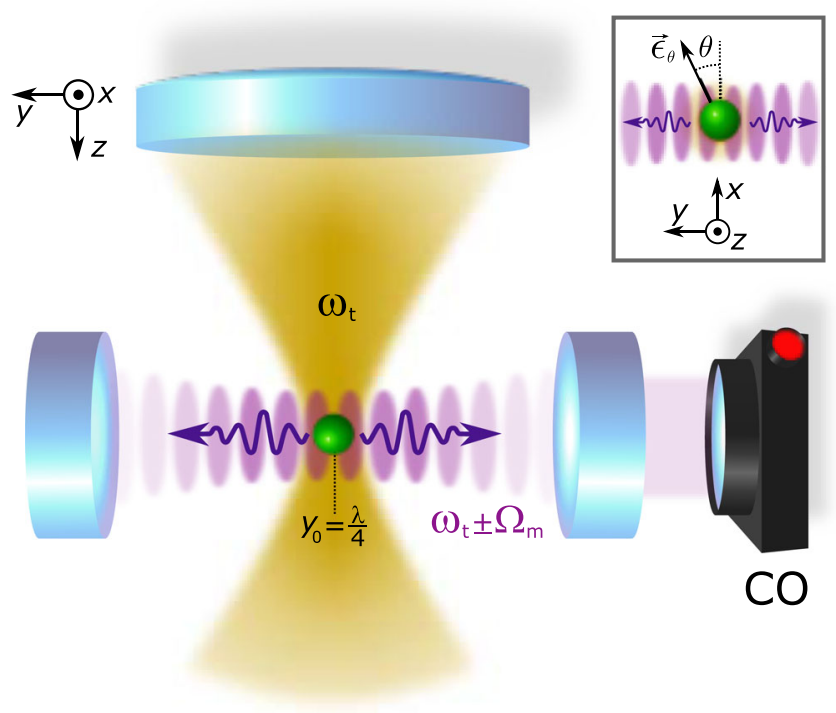

Fig. 1 Experimental setup. An optical tweezers trap (yellow) levitates a silica nanoparticle inside a high finesse cavity. The trapping field is locked relatively to the cavity resonance $\omega_{\text {cav }}$ using Pound-Drever-Hall locking with a detuning $\Delta=\omega_{\mathrm{t}}-\omega_{\text {cav }}$. A 3D piezo stage positions the particle precisely inside the cavity at variable $y_{0}$. The inset displays the linear trap polarisation axis along $\epsilon_{\theta}=\epsilon_{x} \cos \theta$. The rate of coherently scattered photons into the cavity mode (purple) depends on $y_{0}, \epsilon_{\theta}$ and $\Delta$. The transmitted cavity output field is monitored on a photodiode ( $\mathrm{CO}$ ) and the forward scattered trapping light is used to detect the particle motion (see "Experimental setup" section)

interacting with the trapping light, Raman scatters photons into free space and the energy difference between incident and emitted light equals $\pm \hbar \Omega_{m}$ with $m=x, y, z$. In this case, photon up and down conversion are equally probable ${ }^{40}$. The presence of an optical cavity alters the density of states of electromagnetic modes and enhances the CS into the cavity modes through the Purcell effect. If trap photons are red (blue) detuned with respect to the cavity resonance, the cavity enhances photon up (down) conversion and net cooling (heating) takes place.

Coherent scattering theory. In order to estimate the corresponding optomechanical coupling strength in CS, we follow ref. ${ }^{36}$. The interaction Hamiltonian for a polarisable particle interacting with an electric field $\mathbf{E}(\mathbf{R})$ is given by $\hat{\mathrm{H}}_{\mathrm{int}}=-\frac{1}{2} \alpha \mathbf{E}^{2}(\mathbf{R})$ with the particle polarizability $\alpha=4 \pi \epsilon_{0} R^{3} \frac{n_{\mathrm{r}}^{2}-1}{n_{\mathrm{r}}^{2}+2}$ and vacuum permittivity $\epsilon_{0}$. The total electric field consists of the trap $\left(\mathbf{E}_{\mathrm{tr}}(\mathbf{R})\right)$, cavity $\left(\mathbf{E}_{\mathrm{cav}}(\mathbf{R})\right)$ and free space electromagnetic modes $\left(\mathbf{E}_{\text {free }}(\mathbf{R})\right)$ yielding the interaction Hamiltonian

$$
\begin{aligned}
\hat{\mathrm{H}}_{\mathrm{int}} & =-\frac{1}{2} \alpha\left[\mathbf{E}_{\mathrm{tr}}(\mathbf{R})+\mathbf{E}_{\mathrm{cav}}(\mathbf{R})+\mathbf{E}_{\mathrm{free}}(\mathbf{R})\right]^{2} \\
& \approx \hat{\mathrm{H}}_{\mathrm{CS}}+\hat{\mathrm{H}}_{\mathrm{DR}}+\hat{\mathrm{H}}_{\mathrm{CAV}}
\end{aligned}
$$

where $\mathbf{E}_{\text {cav }}(\mathbf{R})$ and $\mathbf{E}_{\text {free }}(\mathbf{R})$ are only populated by scattering events from the particle $\left(n_{\text {trap }} \gg n_{\text {cav }}\right.$ with $n_{\text {trap }}\left(n_{\text {cav }}\right)$ being the number of trap (cavity) photons). As can be seen from Eq. (1), the interaction Hamiltonian consists of six terms of which only the two terms proportional to $\mathbf{E}_{\mathrm{tr}}(\mathbf{R}) \mathbf{E}_{\mathrm{cav}}(\mathbf{R})$ and $\mathbf{E}_{\mathrm{cav}}(\mathbf{R})^{2}$ are relevant for the following discussion ${ }^{36}$. The former one gives rise to the optomechanical coupling by CS, and the latter to the coupling achieved by actively driving the cavity. The term $\propto \mathbf{E}_{\mathrm{tr}}^{2}(\mathbf{R})$ gives rise to the trapping potential, while the term $\propto \mathbf{E}_{\text {tr }}(\mathbf{R}) \mathbf{E}_{\text {free }}(\mathbf{R})$ causes recoil 
heating 36,41 , which can be neglected for the moderate vacuum conditions presented here ${ }^{7,41}$. The remaining two terms can be safely neglected according to ref. ${ }^{36}$.

In the following, we use the simplified interaction Hamiltonian given by Eq. (2) where we separate the parts contributing to the optomechanical coupling due to $\mathrm{CS} \hat{\mathrm{H}}_{\mathrm{CS}}$, active driving $\hat{\mathrm{H}}_{\mathrm{DR}}$, and population of the intracavity field $\hat{\mathrm{H}}_{\mathrm{CAV}}$ (see "Interaction Hamiltonian and power spectral densities" section).

For the measurements presented here, the trap is $x$-polarised with $\theta=0$ (see inset Fig. 1). This simplifies $\hat{\mathrm{H}}_{\mathrm{CS}}$ to $\hat{\mathrm{H}}_{\mathrm{CS}}=-\hbar\left[g_{y}\left(\hat{a}^{\dagger}+\hat{a}\right)\left(\hat{b}_{y}^{\dagger}+\hat{b}_{y}\right)+g_{z}\left(\hat{a}^{\dagger}-\hat{a}\right)\left(\hat{b}_{z}^{\dagger}+\hat{b}_{z}\right)\right]$, where $\hat{a}$ $\left(\hat{a}^{\dagger}\right)$ is the photon annihilation (creation) operator and $\hat{b}\left(\hat{b}^{\dagger}\right)$ is the phonon annihilation (creation) operator. The CS optomechanical coupling strengths $g_{y, z}$ are

$$
\left[\begin{array}{l}
g_{y} \\
g_{z}
\end{array}\right]=\frac{1}{2}\left[\begin{array}{r}
G_{\perp} k_{\mathrm{c}} y_{\mathrm{zpf}} \sin \phi \\
-i G_{\perp} k_{\mathrm{t}} z_{\mathrm{zpf}} \cos \phi
\end{array}\right]
$$

with cavity wavevector $k_{\mathrm{c}}=2 \pi / \lambda_{\mathrm{c}}$, zero-point fluctuations $y_{\text {zpf }}, z_{\text {zpf }}=\sqrt{\frac{\hbar}{2 m \Omega_{y, z}}}$ and $\phi=2 \pi y_{0} / \lambda_{\mathrm{c}}$, with $y_{0}$ being the particle position along the cavity axis and $y_{0}=\lambda_{\mathrm{c}} / 4$ corresponding to the intensity minimum.

The optical cavity resonance frequency shift caused by a particle located at maximum intensity of the intracavity standing wave is $G_{\perp}=\alpha E_{0} \sqrt{\frac{\omega_{c}}{2 \hbar \epsilon_{0} V_{c}}}$ with cavity mode volume $V_{\mathrm{c}}=\pi \mathrm{w}_{\mathrm{c}}^{2} L_{\mathrm{c}} / 4$, cavity waist $w_{\mathrm{c}}$, cavity length $L_{\mathrm{c}}$, and $\omega_{\mathrm{c}}=2 \pi \mathrm{c} / \lambda_{\mathrm{c}}$ The trap electric field is $E_{0}=\sqrt{\frac{4 P_{\mathrm{t}}}{\pi \epsilon_{0} c \mathrm{w}_{x} \mathrm{w}_{y}}}$ with trap waists $w_{x}$ and $w_{y}$.

Due to the intracavity standing wave, the optomechanical coupling strength has a sinusoidal dependence on $y_{0}$ with opposite phase for $g_{y}$ and $g_{z}$. In contrast, $g_{x}=0$ if $\theta=0$.

For clarity, we limit the discussion to coupling along the cavity axis $(y)$, such that $\Omega_{m}=\Omega_{y}$ and $g=g_{y}$. Similar results can be obtained for the other directions $x, z$ with the same level of control.

The maximum expected coupling strength from CS is $g_{y}^{\max }=$ $G_{\perp} k_{\mathrm{c}} y_{\text {zpf }}=2 \pi \times 31.7 \mathrm{kHz}$ for our parameters. However, we displace the particle by $\delta z \approx 40 \mu \mathrm{m}$ from the cavity centre for better experimental stability. Hence, our expected optomechanical coupling strength is reduced by $\approx 30 \%$ down to $g_{y}^{\text {th }}=2 \pi \times 22.6 \mathrm{kHz}$, enabling the SCR with $g_{y}>\kappa$. Despite the fact that this value is a factor of $\approx 3$ lower than previously reported ${ }^{9}$, the deep SRC with $g>\kappa$ remains unaccomplished.

Transition to the SCR. In the weak coupling regime $g<\kappa$, the Lorentzian-shaped spectra of our mechanical oscillator displays a single peak at its resonance frequency $\Omega_{m}$. When $g$ increases, the energy exchange rate between optical and mechanical mode grows until the SCR is reached at $g>\kappa / 4$ (ref. ${ }^{33}$ ). In the SCR, the optical and mechanical mode hybridise, which gives rise to two new eigenmodes at shifted eigenfrequencies $\Omega_{ \pm}$(see Eq. (9)). At this point, the energy exchange in between the optical and mechanical mode is faster than the decoherence rate of each individual mode. The hybridised eigenmode frequencies

$$
\Omega_{ \pm}=\Omega_{m}-\frac{\Omega_{m}+\Delta}{2} \pm \sqrt{g_{y}^{2}+\left(\frac{\Omega_{m}+\Delta}{2}\right)^{2}}
$$

experience an avoided crossing, the so-called NMS, which reaches a maximum of $\Omega_{+}-\Omega_{-}=2 g_{y}$ at the optimal detuning $\Delta=-\Omega_{m}$. The linewidth of the hybrid modes at this detuning is $\left(\kappa+\Gamma_{\mathrm{m}}\right) / 2$.
Therefore, $\Gamma_{\mathrm{m}}$ needs to be smaller or comparable to $\kappa$ to resolve the NMS of $2 g_{y}$.

As can be seen from Eq. (3), we control $g_{y}$ through various parameters like the trap power $P_{\mathrm{t}}$, the particle position $y_{0}$ and the polarisation angle $\theta$. The optical coupling rate $\Gamma_{\text {opt }}$ depends additionally on the trap detuning $\Delta$ and is maximised at $\Delta=-\Omega_{m}$ to $\Gamma_{\text {opt }}=4 g_{y}^{2} / \kappa$ (refs. $7\left[, 12\right.$ ). While $P_{\mathrm{t}}$ and $\Delta$ only influence the magnitude of the coupling strength, $y_{0}$ and $\theta$ change also the nature of the coupling from $1 \mathrm{D}$ to potentially $3 \mathrm{D}^{36}$. For simplicity, we focus on varying $\Delta$ and $y_{0}$ in the following measurements and keep $P_{\mathrm{t}}, \theta$ and $\Gamma_{\mathrm{m}}=2 \pi \times 0.8 \mathrm{kHz}$, corresponding to $p=1.4 \mathrm{mBar}$, fixed (see "Experimental setup" section). The range of $\Delta$ is limited due to instabilities in the experiment.

Observation of strong coupling. Figure 2 left panel displays the experimental position power spectral density (PSD) versus $\Delta$ for different $y_{0}$. Throughout the remaining part of the manuscript, we fit our PSD to Eq. (9), if not stated differently. From this fit, we can extract the hybridised modes $\Omega_{ \pm}$that are separated by $2 g_{y}$. We cover a total distance of $\delta y_{0} \approx \lambda_{\mathrm{c}} / 4$ and change the optomechanical coupling strength, and therefore also the NMS, from (a) $g_{y} / 2 \pi=22.8 \mathrm{kHz}$, (b) $15.4 \mathrm{kHz}$, (c) $4.6 \mathrm{kHz}$ and (d) $0 \mathrm{kHz}$, exploring the entire range from strong coupling to zero coupling. The right panel shows the fit, which is in good agreement with the data. We observe two eigenmodes $\Omega_{ \pm}$with an exceptional NMS of $2 g_{y} \approx 4.6 \kappa$ at $\Delta=-\Omega_{m}$, corresponding to $20 \%$ of the bare mechanical eigenfrequency, once the system enters the SCR at $g>$ $\kappa / 4$ (ref. ${ }^{33}$ ). For $g_{y}=0$, we observe only the mechanical mode with slightly increased frequency $\Omega_{m}=2 \pi \times 200 \mathrm{kHz}$ due to the additional trapping potential supplied by the cavity field (see Fig. 2d). In Fig. 2a and b, we observe an additional NMS in the $y$-mode, which stems from a second cross polarised optical mode. Note that, throughout all our measurements (see Figs. 2-4), the second NMS is the largest source for discrepancies between experiment and theory (for more details, see Supplementary Information). We also attribute the NMS of the $x$-mode at $\Omega / \Omega_{m}=0.89$ to the second optical mode as observed in Fig. 2 d, since the $x$-mode should be decoupled from the first mode $\left(g_{x}=0\right.$ if $\theta=0)$.

Figure $3 \mathrm{a}-\mathrm{c}$ displays the particle's position PSD at different $\Delta$ while it is located at the intensity minimum, corresponding to the position of maximum coupling $g_{y}=2.3 \kappa$, displayed in Fig. 2a. Our theory (yellow) captures the data (purple) well. In Fig. $3 a$, the optical mode and mechancial mode begin to hybridise into new eigenmodes at $\Delta=-1.5 \Omega_{m}$ which is confirmed by a second peak appearing at $\Omega \approx 2 \pi \times 300 \mathrm{kHz}$. The hybridisation becomes stronger as $\Delta$ approaches the cavity resonance and the NMS is maximised at $\Delta \approx-\Omega_{m}$ as shown in Fig. 3c. The dependence of the new eigenmodes $\Omega_{ \pm}$on $\Delta$ is shown in Fig. $3 \mathrm{~d}$, displaying clearly the expected avoided crossing of $2 g_{y}$. The solid line is a fit to Eq. (4). The edges of the shaded area represent the upper and lower limit of the fit, which we obtain by fitting only the upper branch (yellow) or the lower branch (purple), respectively.

As already discussed previously, our experiment allows to change the optomechanical coupling by changing various experimental parameters, which stands in contrast to many other experimental platforms. Figure 4 displays this flexibility to reach the SCR by demonstrating the position dependence of $g y$ at optimal detuning $\Delta \approx-\Omega_{m}$ extracted from the data Fig. $2 \mathrm{a}-\mathrm{d}$. The experimental and theoretical position PSDs versus $y_{0}$ are depicted in Fig. $4 \mathrm{a}$ and b. The mode at $\Omega / \Omega_{m} \approx 0.89$ corresponds to the decoupled $x$-mode. The dashed line highlights the theoretical frequency of the eigenmodes $\Omega_{ \pm} / \Omega_{m}$ following Eq. (4). In both experiment and theory, we observe the expected sinusoidal behaviour predicted by Eq. (3). Figure $4 \mathrm{c}$ depicts 


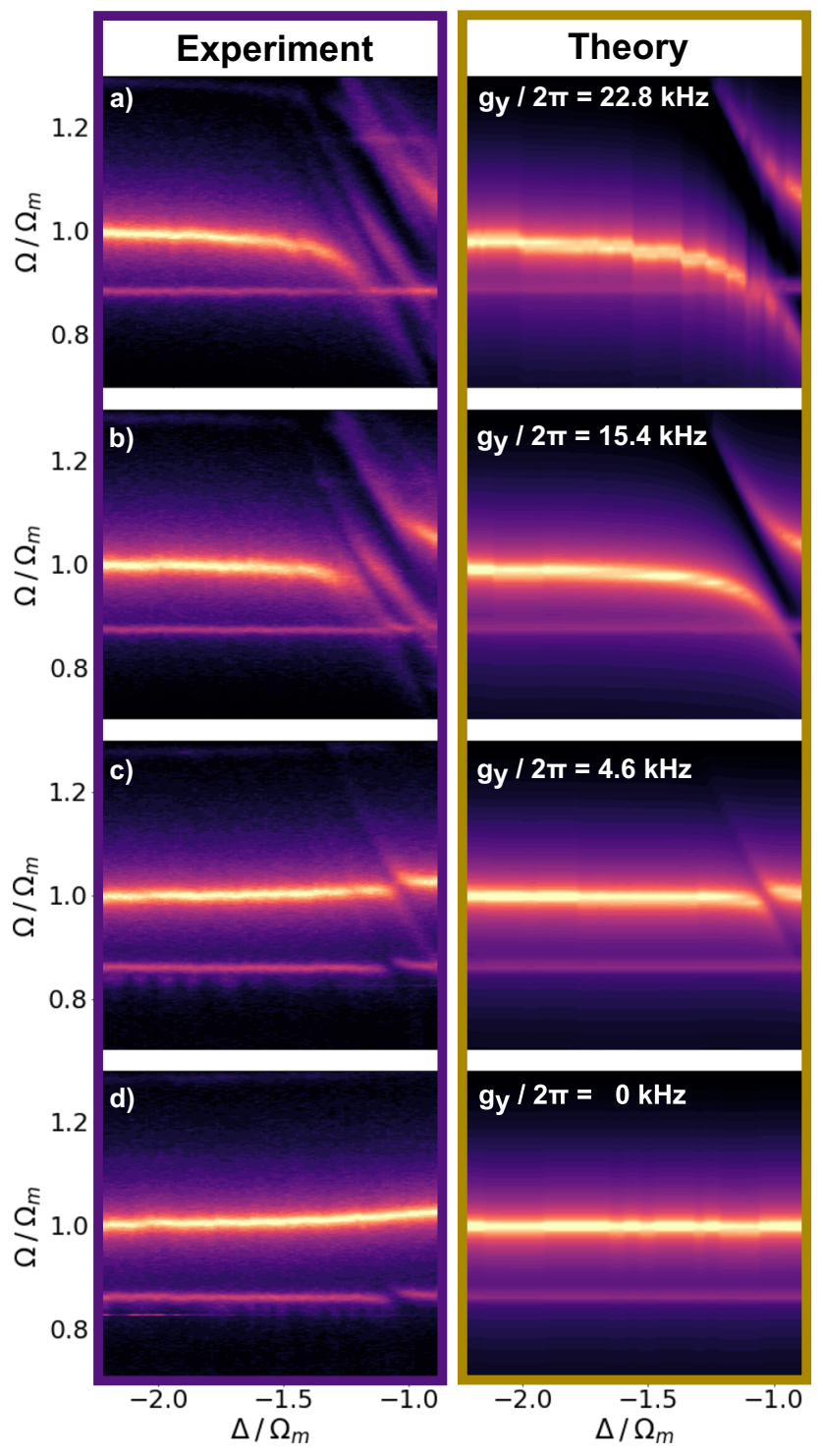

Fig. 2 Normal mode splitting. Particle's position power spectral density $\operatorname{PSD}(\Omega)$ versus $\Delta$ for different $y_{0}$ and therefore various $g_{y}$. Experimental data are displayed on the left, and theory on the right. The bare mechanical (optical) modes correspond to horizontal (diagonal) lines. a Maximum normal mode splitting of $2 g_{y}$ is observed at $\Delta=-\Omega_{m}$ yielding a value of $g_{y}=2 \pi \times 22.8 \mathrm{kHz}=2.3 \kappa$, where $y_{0} \approx \lambda_{c} / 4$ is close to the intensity minimum (see Eq. (3)). b When the particle is moved by $\delta y_{0} \approx 0.12 \lambda_{c}$, the coupling reduces to $g_{y}=2 \pi \times 15.4 \mathrm{kHz}=1.5 \kappa$. c Normal mode splitting is still visible at $\delta y_{0} \approx 0.2 \lambda_{c}$, yielding $g_{y}=2 \pi \times 4.6 \mathrm{kHz}=$ $0.46 \kappa$. $\mathbf{d}$ At the intensity maximum, corresponding to a shift of $\delta y_{0} \approx \lambda_{c} / 4$ and $g_{y}=0 \mathrm{kHz}$, the normal mode splitting vanishes and we only see a shift of $\delta \Omega_{m} \approx 2 \pi \times 5 \mathrm{kHz}$ in the mechanical frequency due to the increased intracavity photon number (see Supplementary Fig. 1). In general, we observe a good agreement between experimental data and theory. We attribute discrepancies to a second cross polarised cavity mode inducing a second normal mode splitting (for more details, see Supplementary Information).

$\left|g_{y}\right|=\left(\Omega_{+}-\Omega_{-}\right) / 2$ (circles) extracted from Fig. 4a. The dashed line represents the fit to the absolute value of Eq. (3) yielding $g_{y}^{\exp }=2 \pi \times(22.8 \pm 0.2) \mathrm{kHz}$ which coincides well with the theoretical value of $g_{y}^{\text {th }}=2 \pi \times 22.6 \mathrm{kHz}$. The measured period coincides with the expected period of $\lambda_{\mathrm{c}} / 4$. The shaded area corresponds to $3 \sigma_{\text {std }}$ of the fit.

\section{Discussion}

As a figure of merit to assess the potential of our system for quantum applications, we use the quantum cooperativity, which yields here $C_{\mathrm{CS}}=\left(2 g_{y}^{\max }\right)^{2} /\left(\kappa \Gamma_{\mathrm{m}}\left(n_{\mathrm{th}}+1\right)\right)=8 \times 10^{-6}$ at a pressure $p=1.4 \mathrm{mbar}$ and promises a value as large as $C_{\mathrm{CS}} \approx 36$ at $p=3 \times 10^{-7}$ mbar, since $\Gamma_{\mathrm{m}} \propto p$. At this low pressure, the photon recoil heating rate $\Gamma_{\text {rec }}{ }^{41}$ equals our mechanical decoherence rate $\left.\Gamma_{\mathrm{m}}\left(n_{\mathrm{th}}+1\right)\right)$, and therefore halves the reachable $C_{\mathrm{CS}}$. The maximum $C_{\mathrm{CS}}$ is ultimately limited by $\Gamma_{\mathrm{rec}}$, regardless if we reduce the pressure even further. Nevertheless, this predicted value of $C_{\mathrm{CS}}$ is many orders of magnitude larger than what has been achieved in levitation setups by actively driving the cavity ${ }^{7,24}$ and larger than achieved in ref. ${ }^{9}$. More importantly it enables coherent quantum control at $g \gg \kappa, \Gamma_{\mathrm{m}} \cdot n_{\text {th }}$ at pressure levels $p \leq 10^{-6}$ mbar, a pressure regime commonly demonstrated in numerous levitation experiments ${ }^{7,9,41}$.

Furthermore, our experimental parameters promise the possibility of motional ground state cooling in our system ${ }^{9}$, which in combination with coherent quantum control enables us to fully enter the quantum regime with levitated systems and to create non-classical states of motion and superposition states of macroscopic objects in free fall experiments ${ }^{10,11}$ in the future.

\section{Methods}

Experimental setup. The experimental setup is displayed in Fig. 5. A silica nanoparticle is loaded at ambient pressure into a long range single beam trap and transferred to a more stable, short range optical tweezers trap ${ }^{42}$ (with wavelength $\lambda_{\mathrm{t}}=1064 \mathrm{~nm}$, power $P \simeq 150 \mathrm{~mW}$, focusing lens $\mathrm{NA}=0.8$ ) inside a vacuum chamber. Due to the tight focusing, the nanoparticle non-degenerate eigenfrequencies are $\Omega_{x, y, z}=2 \pi \times(172 \mathrm{kHz}, 197 \mathrm{kHz}, 56 \mathrm{kHz})$, respectively. The optical tweezers are mounted on a $3 \mathrm{D}$ nanometre resolution piezo system allowing for precise $3 \mathrm{D}$ positioning inside a high finesse Fabry-Perot cavity (with cavity finesse $F=540,000$, free spectral range FSR $=2 \pi \times 5.4 \mathrm{GHz}$ )

In order to control the detuning $\Delta=\omega_{\mathrm{t}}-\omega_{\mathrm{c}}$ between the cavity resonance $\omega_{c}$ and the trap field $\omega_{\mathrm{t}}$, we use a weak cavity field for locking the cavity via the PoundDrever-Hall technique (PDH) on the TEM01 mode minimising additional heating effects through the photon recoil heating of the cavity lock field. The PDH errorsignal acts on the internal laser piezo and an external AOM (not shown). We separate lock and trap light in frequency space by one free spectral range (FSR) such that the total detuning between lock and trap yields $\omega_{\mathrm{t}}=\omega-\mathrm{FSR}-\Delta$. The variable EOM modulation $F S R+\Delta$ is provided by a signal generator. The intracavity power can be deduced from the transmitted cooling light observed on a photodiode behind the cavity (CO).

All particle information shown is gained in forward balanced detection interfering the scattered light field and the non-interacting part of the trap beam as shown in Fig. 5. The highly divergent trap light is collected using a lens (NA $=0.8$ ). We use three balanced detectors (FS) to monitor the oscillation of the particle in all three degrees of freedom.

The data time traces are acquired at $1 \mathrm{MHz}$ acquisition rate. Each particle position PSD is obtained by averaging over $N=25$ samples of which each one is calculated from individual $40 \mathrm{~ms}$ time traces, corresponding to a total measurement time of $t=1 \mathrm{~s}$.

We keep the pressure stable at $p=1.4$ mbar. The thermal bath couples as

$$
\Gamma_{\mathrm{m}}=\frac{k_{\mathrm{B}} T}{\hbar \mathrm{Q}_{\mathrm{m}} n_{\mathrm{th}}}=15.8 \frac{R^{2} p}{m v_{\text {gas }}}
$$

where $Q_{\mathrm{m}}=\Omega_{m} / \Gamma_{\mathrm{m}}$ is the mechanical quality factor, $n_{\mathrm{th}}=\frac{k_{\mathrm{B}} T}{\hbar \Omega_{m}}$ the thermal occupation number, $R$ the particle radius, $p$ the surrounding gas pressure and $v_{\text {gas }}=\sqrt{3 k_{\mathrm{B}} T / m_{\mathrm{gas}}}$.

In the measurements presented, we cool our particle's centre of mass motion to $T=235$, corresponding to a reduction of the phonon occupation by roughly $20 \%$. The theoretically expected heating rate due to the residual gas accounts fully for the experimentally observed heating rate.

Interaction Hamiltonian and power spectral densities. Following ref. ${ }^{36}$, the relevant contributions to the CS interaction Hamiltonian for $\theta=0$ are given by

$$
\begin{aligned}
\frac{\hat{\mathrm{H}}_{\mathrm{CS}}}{\hbar}= & -g_{y}\left(\hat{a}^{\dagger}+\hat{a}\right)\left(\hat{b}_{y}^{\dagger}+\hat{b}_{y}\right) \\
& -g_{z}\left(\hat{a}^{\dagger}-\hat{a}\right)\left(\hat{b}_{z}^{\dagger}+\hat{b}_{z}\right)
\end{aligned}
$$



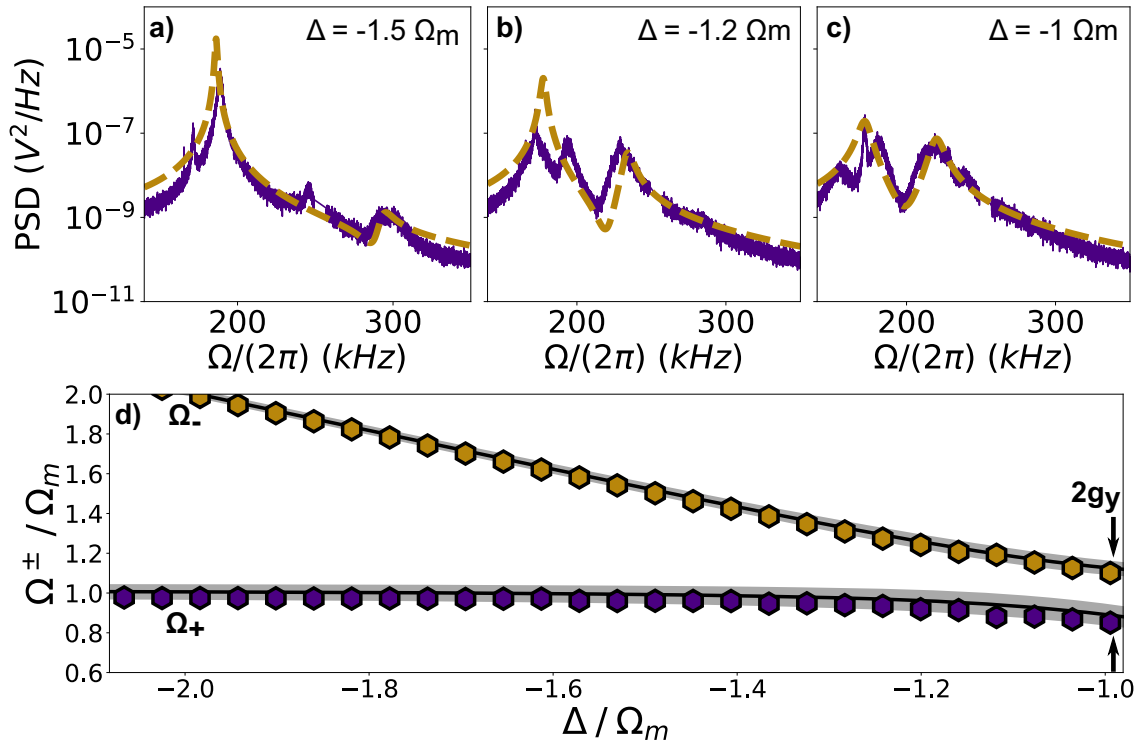

Fig. 3 Power spectral density versus cavity detuning $\Delta$. a-c Experiment (purple) and theory (yellow, dashed) fitted to Eq. (9) at $\Delta=-2 \pi \times 293 \mathrm{kHz} \approx$ $-1.5 \Omega_{m}(\mathbf{a}), \Delta=-2 \pi \times 225 \mathrm{kHz} \approx-1.2 \Omega_{m}$ (b) and $\Delta=-2 \pi \times 205 \mathrm{kHz} \approx-\Omega_{m}$ (c). The optomechanical coupling strength $g_{y}$ grows with increasing $\Delta$. Optical and mechanical modes start to hybridise clearly at $\Delta \geq-1.5 \Omega_{m}$. We attribute the discrepancy between data and theory to the second optical mode (see Supplementary Informtation). $\mathbf{d}$ Hybridised eigenmodes $\Omega_{ \pm}$versus $\Delta$ at the intensity minimum $\left(y_{0} \approx \lambda_{d} / 4\right)$. Maximum normal mode splitting of $2 g_{y}$ with $g_{y}=2 \pi \times 22.8 \mathrm{kHz}=2.3 \kappa$ occurs at $\Delta=-\Omega_{m}$. The black line fits the data to Eq. (4), while the inner (outer) edges of the grey area correspond to a fit using solely to the upper (lower) branch $\Omega_{-}\left(\Omega_{+}\right)$.
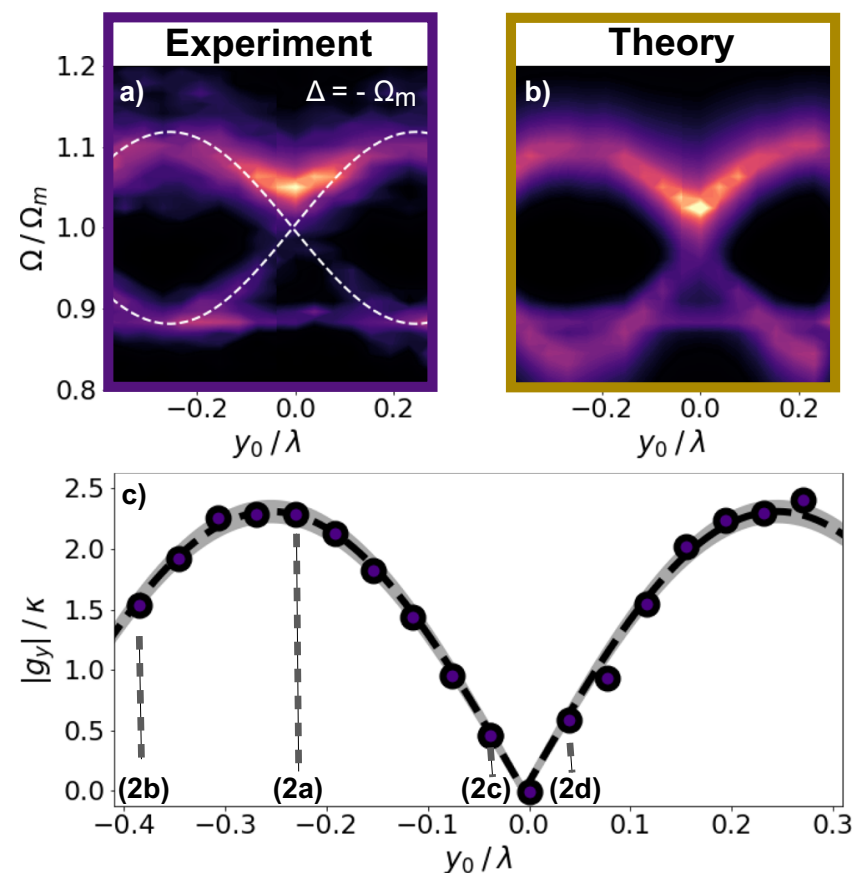

Fig. 4 Normal mode splitting versus particle position $\mathbf{y}_{\mathbf{0}}$. a Experiment and $\mathbf{b}$ theory according to Eq. (9). Particle position power spectral density $\operatorname{PSD}(\Omega)$ at the optimal $\Delta=-2 \pi \times 193 \mathrm{kHz} \approx-\Omega_{m}$ along $y_{0}$ is shown. The hybridised modes split by $2 g_{y}$. The white dashed line displays $\Omega_{ \pm} / \Omega_{m}=1 \pm$ $g_{y} / \Omega_{m}$ where $g_{y}$ follows Eq. (3). The mechanical mode at $\Omega / \Omega_{m} \approx 0.89$ corresponds to the mechanical $x$-mode. The data and fit show very good agreement. $\mathbf{c}\left|g_{y}\right|$ at $\Delta \approx-\Omega_{m}$ versus $y_{0}$. Maximum and minimum coupling are separated by $\delta y_{0}=\lambda_{c} / 4$ as expected by Eq. (3). Black dashed line fits to the absolute value of Eq. (3) with a maximum $g_{y}^{\max } \approx-2.3 \kappa$ and the grey shaded area corresponds to $3 \sigma_{\text {std }}$ of the fit. The dotted lines indicate the positions used in Fig. 2.

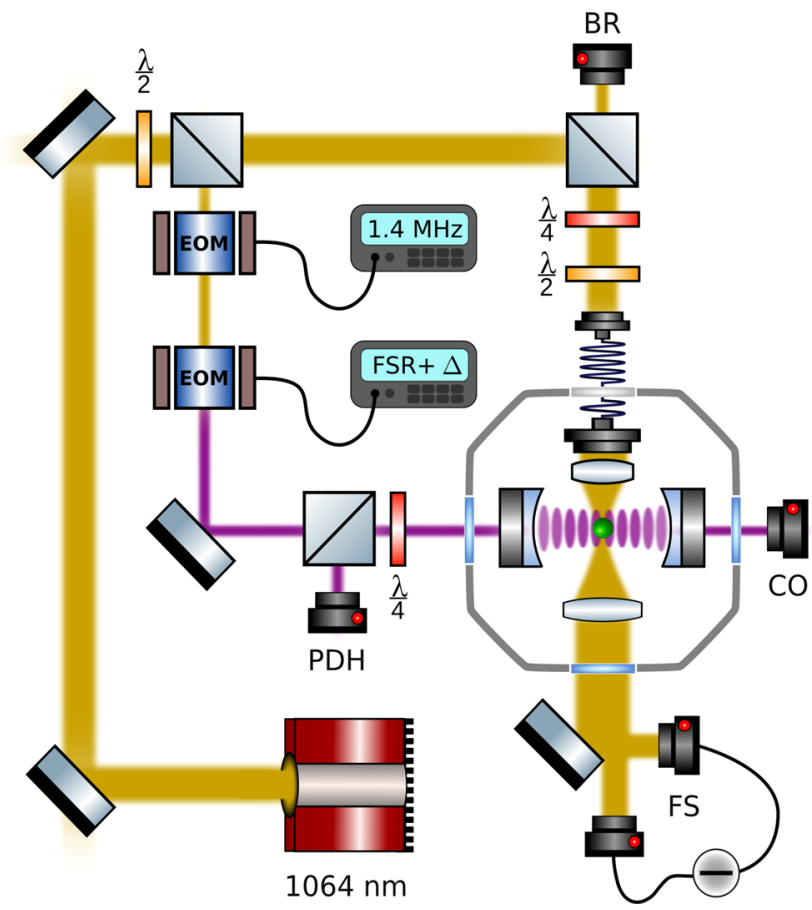

Fig. 5 Extended experimental setup. A $1064 \mathrm{~nm}$ Mephisto laser (yellow) traps a silica particle of $d=177 \mathrm{~nm}$ inside a high finesse cavity (purple). The trap light is locked at a variable detuning $\Delta+$ FSR from the cavity resonance via the Pound-Drever-Hall technique by detecting the error signal on a photodiode (PDH). The particle motion is detected in backreflection (BR) and balanced forward detection (FS). The intracavity field is estimated from the transmitted power detected on a photodiode (CO). 


$$
\begin{aligned}
& \frac{\hat{\mathrm{H}}_{\mathrm{DR}}}{\hbar}=-g_{y}^{\mathrm{dr}} \hat{a}^{\dagger} \hat{a}\left(\hat{b}_{y}^{\dagger}+\hat{b}_{y}\right) \\
& \frac{\hat{\mathrm{H}}_{\mathrm{CAV}}}{\hbar}=-\frac{G_{\perp}}{2}\left(\hat{a}^{\dagger}+\hat{a}\right) \cos \phi
\end{aligned}
$$

The single photon optomechanical coupling strength achieved by actively driving the cavity is $g_{y}^{\mathrm{dr}}=\frac{\alpha \omega_{\mathrm{c}}}{2 \epsilon_{0} V_{\mathrm{c}}} k_{\mathrm{c}} y_{\mathrm{zpf}} \sin (2 \phi)=2 \pi \times 0.05 \mathrm{~Hz} \sin (2 \phi)$. This value is enhanced by the intracavity photon number $n_{\text {cav }}=1.6 \times 10^{8}$, inferred from the transmitted cavity power $P_{\text {out }}$. At optimal conditions, we achieve $g_{y}^{\mathrm{rp}} \sqrt{n_{\mathrm{c}}}=$ $2 \pi \times 0.6 \mathrm{kHz}$. Thus, the optomechanical coupling strength is about 40 times larger for CS, since the photons contributing to the CS interaction are confined in a much smaller volume due to the much smaller trap waist $w_{\mathrm{t}} \times w_{\mathrm{c}} \ll w_{\mathrm{c}}^{2}$.

The mechanical susceptibility $\chi$ is given as ${ }^{33}$

$$
\begin{aligned}
& |\chi(\Omega)|^{2}=\frac{1}{m^{2}\left[\left(\Omega_{m}^{2}+2 \Omega \delta \Omega_{m}(\Omega)-\Omega^{2}\right)^{2}+\left(\Omega \Gamma_{\text {eff }}(\Omega)\right)^{2}\right]} \\
& \Gamma_{\text {eff }}(\Omega)=\Gamma_{\mathrm{m}}+\Gamma_{\text {opt }}(\Omega) \\
& \delta \Omega_{m}(\Omega)=g_{y}^{2} \frac{\Omega_{m}}{\Omega}\left[\frac{\Delta+\Omega}{(\Delta+\Omega)^{2}+\kappa^{2} / 4}+\frac{\Delta-\Omega}{(\Delta-\Omega)^{2}+\kappa^{2} / 4}\right] \\
& \Gamma_{\text {opt }}(\Omega)=g_{y}^{2} \frac{\Omega_{m}}{\Omega}\left[\frac{\kappa}{(\Delta+\Omega)^{2}+\kappa^{2} / 4}-\frac{\kappa}{(\Delta-\Omega)^{2}+\kappa^{2} / 4}\right]
\end{aligned}
$$

with the effective (optical) damping $\Gamma_{\text {eff }}\left(\Gamma_{\text {opt }}\right)$ and the optomechanical spring effect $\delta \Omega_{m}$. We fit the three mechanical modes $\Omega_{x, y, z}$ to Eq. (9) where $g_{y}, \kappa, \Gamma_{\mathrm{m}}$ and the relative mode amplitudes are chosen as free fit parameters.

\section{Data availability}

The data that support the findings of this study are available from the corresponding author upon request.

Received: 29 May 2020; Accepted: 26 November 2020; Published online: 12 January 2021

\section{References}

1. Chang, D. E. et al. Cavity opto-mechanics using an optically levitated nanosphere. Proc. Natl Acad. Sci. USA 07, 1005-1010 (2010).

2. Romero-Isart, O., Juan, M. L., Quidant, R. \& Cirac, J. I. Toward quantum superposition of living organisms. N. J. Phys. 12, 033015 (2010).

3. Kiesel, N. et al. Cavity cooling of an optically levitated submicron particle. Proc. Natl Acad. Sci. USA 110, 35 (2013).

4. Asenbaum, P., Kuhn, S., Nimmrichter, S., Sezer, U. \& Arndt, M. Cavity cooling of free silicon nanoparticles in high vacuum. Nat. Commun. 4, 2743 (2013).

5. Millen, J., Fonseca, P. Z. G., Mavrogordatos, T., Monteiro, T. S. \& Barker, P. F. Cavity cooling a single charged levitated nanosphere. Phys. Rev. Lett. 114 123602 (2015).

6. Fonseca, P. Z. G., Aranas, E. B., Millen, J., Monteiro, T. S. \& Barker, P. F. Nonlinear dynamics and strong cavity cooling of levitated nanoparticles. Phys. Rev. Lett. 117, 173602 (2016)

7. Meyer, N. et al. Resolved-sideband cooling of a levitated nanoparticle in the presence of laser phase noise. Phys. Rev. Lett. 123, 1-6 (2019).

8. Delić, U. et al. Cavity cooling of a levitated nanosphere by coherent scattering. Phys. Rev. Lett. 122, 123602 (2019).

9. Delić, U. et al. Cooling of a levitated nanoparticle to the motional quantum ground state. Science 367, 892-895 (2020).

10. Romero-Isart, O. et al. Large quantum superpositions and interference of massive nanometer-sized objects. Phys. Rev. Lett. 107, 020405 (2011).

11. Bateman, J., Nimmrichter, S., Hornberger, K. \& Ulbricht, H. Near-field interferometry of a free-falling nanoparticle from a point-like source. Nat. Commun. 5, 1-5 (2014).

12. Aspelmeyer, M., Kippenberg, T. J. \& Marquardt, F. Cavity optomechanics. Rev. Mod. Phys. 86, 1391-1452 (2014).

13. Millen, J., Monteiro, T. S., Pettit, R. \& Vamivakas, A. N. Optomechanics with levitated particles. Rep. Prog. Phys. 83, 026401 (2020).

14. Hempston, D. et al. Force sensing with an optically levitated charged nanoparticle. Appl. Phys. Lett. 111, 133111 (2017).

15. Arita, Y., Mazilu, M. \& Dholakia, K. Laser-induced rotation and cooling of a trapped microgyroscope in vacuum. Nat. Commun. 4, 2374 (2013).
16. Kuhn, S. et al. Optically driven ultra-stable nanomechanical rotor. Nat. Commun. 8, 1670 (2017).

17. Monteiro, F., Ghosh, S., van Assendelft, E. C. \& Moore, D. C. Optical rotation of levitated spheres in high vacuum. Phys. Rev. A 97, 051802(R) (2018).

18. Reimann, R. et al. GHz rotation of an optically trapped nanoparticle in vacuum. Phys. Rev. Lett. 121, 033602 (2018).

19. Hebestreit, E., Frimmer, M., Reimann, R. \& Novotny, L. Sensing static forces with free-falling nanoparticles. Phys. Rev. Lett. 121, 063602 (2018).

20. Rondin, L. et al. Direct measurement of Kramers turnover with a levitated nanoparticle. Nat. Nanotechnol. 12, 1130-1134 (2017).

21. Marshall, W., Simon, C., Penrose, R. \& Bouwmeester, D. Towards quantum superpositions of a mirror. Phys. Rev. Lett. 91, 13 (2003).

22. Kleckner, D. et al. Creating and verifying a quantum superposition in a microoptomechanical system. N. J. Phys. 10, 095020 (2008).

23. Windey, D. et al. Cavity-based 3D cooling of a levitated nanoparticle via coherent scattering. Phys. Rev. Lett. 122, 123601 (2019).

24. Delić, U. et al. Levitated cavity optomechanics in high vacuum. Quantum Sci. Technol. 5, 025006 (2020).

25. Safavi-Naeini, A. H. et al. Squeezed light from a silicon micromechanical resonator. Nature 500, 185-189 (2013).

26. Riedinger, R. et al. Non-classical correlations between single photons and phonons from a mechanical oscillator. Nature 530, 313-316 (2016).

27. Riedinger, R. et al. Remote quantum entanglement between two micromechanical oscillators. Nature 556, 473-477 (2018).

28. Chen, J., Rossi, M., Mason, D. \& Schliesser, A. Entanglement of propagating optical modes via a mechanical interface. Nat. Commun. 11, 943 (2020).

29. Gröblacher, S., Hammerer, K., Vanner, M. R. \& Aspelmeyer, M. Observation of strong coupling between a micromechanical resonator and an optical cavity field. Nature 460, 724-727 (2009).

30. Teufel, J. D. et al. Circuit cavity electromechanics in the strong-coupling regime. Nature 471, 204-208 (2011)

31. Teufel, J. D. et al. Sideband cooling of micromechanical motion to the quantum ground state. Nature 475, 359-363 (2011).

32. Verhagen, E., Deléglise, S., Weis, S., Schliesser, A. \& Kippenberg, T. J. Quantum-coherent coupling of a mechanical oscillator to an optical cavity mode. Nature 482, 63-67 (2012).

33. Dobrindt, J. M., Wilson-Rae, I. \& Kippenberg, T. J. Parametric normal-mode splitting in cavity optomechanics. Phys. Rev. Lett. 101, 1-4 (2008).

34. Thompson, R. J., Rempe, G. \& Kimble, H. J. Observation of normal-mode splitting for an atom in an optical cavity. Phys. Rev. Lett. 68, 1132-1135 (1992).

35. Vuletić, V., Chan, H. W. \& Black, A. T. Three-dimensional cavity Doppler cooling and cavity sideband cooling by coherent scattering. Phys. Rev. A 64, 033405 (2001).

36. Gonzalez-Ballestero, C. et al. Theory for cavity cooling of levitated nanoparticles via coherent scattering: master equation approach. Phys. Rev. A 100, 013805 (2019)

37. Chikkaraddy, R. et al. Single-molecule strong coupling at room temperature in plasmonic nanocavities. Nature 535, 127-130 (2016).

38. Kleemann, M. E. et al. Strong-coupling of WSe2 in ultra-compact plasmonic nanocavities at room temperature. Nat. Commun. 8, 1296 (2017).

39. Gieseler, J. Dynamics of Optically Levitated Nanoparticles in High Vacuum. Ph.D. thesis, ICFO (2014).

40. Tebbenjohanns, F., Frimmer, M., Jain, V., Windey, D. \& Novotny, L. Motional sideband asymmetry of a nanoparticle optically levitated in free space. Phys. Rev. Lett. 124, 13603 (2020).

41. Jain, V. et al. Direct measurement of photon recoil from a levitated nanoparticle. Phys. Rev. Lett. 116, 243601 (2016).

42. Mestres, P. et al. Cooling and manipulation of a levitated nanoparticle with an optical fiber trap. Appl. Phys. Lett. 107, 151102 (2015)

\section{Acknowledgements}

The authors thank J. Gieseler and C. González-Ballestero for stimulating discussions. The project acknowledges financial support from the European Research Council through grant QnanoMECA (CoG-64790), Fundació Privada Cellex, CERCA Programme/Generalitat de Catalunya, and the Spanish Ministry of Economy and Competitiveness through the Severo Ochoa Programme for Centres of Excellence in R\&D (SEV-20150522). This project has received funding from the European Union's Horizon 2020 research and innovation programme under the Marie Skłodowska-Curie grant agreement No. 713729.

\section{Author contributions}

A.d.l.R.S. performed the measurements, N.M. evaluated the data and wrote the manuscript, R.Q. supervised the study.

\section{Competing interests}

The authors declare no competing interests. 


\section{Additional information}

Supplementary information is available for this paper at https://doi.org/10.1038/s41467020-20419-2.

Correspondence and requests for materials should be addressed to N.M.

Peer review information Nature Communications thanks James Millen and the other anonymous reviewer(s) for their contribution to the peer review of this work.

Reprints and permission information is available at http://www.nature.com/reprints

Publisher's note Springer Nature remains neutral with regard to jurisdictional claims in published maps and institutional affiliations. (c) (i) Open Access This article is licensed under a Creative Commons Attribution 4.0 International License, which permits use, sharing, adaptation, distribution and reproduction in any medium or format, as long as you give appropriate credit to the original author(s) and the source, provide a link to the Creative Commons license, and indicate if changes were made. The images or other third party material in this article are included in the article's Creative Commons license, unless indicated otherwise in a credit line to the material. If material is not included in the article's Creative Commons license and your intended use is not permitted by statutory regulation or exceeds the permitted use, you will need to obtain permission directly from the copyright holder. To view a copy of this license, visit http://creativecommons.org/ licenses/by/4.0/.

(C) The Author(s) 2021 